\title{
Characterization of Redlen CZT detectors for x-ray astronomy
}

Sean N. Pike, Fiona A. Harrison, Jill A. Burnham, Walter W. Cook, Brian W. Grefenstette, et al.

Sean N. Pike, Fiona A. Harrison, Jill A. Burnham, Walter W. Cook, Brian W. Grefenstette, Kristin K. Madsen, Hiromasa Miyasaka, "Characterization of Redlen CZT detectors for x-ray astronomy," Proc. SPIE 10709, High Energy, Optical, and Infrared Detectors for Astronomy VIII, 107091D (7 August 2018); doi: $10.1117 / 12.2313860$

Event: SPIE Astronomical Telescopes + Instrumentation, 2018, Austin, Texas, United States 


\title{
Characterizing Redlen CZT detectors for X-ray astronomy
}

\author{
Sean N. Pike, Fiona A. Harrison, Jill A. Burnham, Walter W. Cook, Brian W. Grefenstette, \\ Kristin K. Madsen, and Hiromasa Miyasaka \\ Space Radiation Laboratory, California Institute of Technology, Pasadena, CA, 91125
}

\begin{abstract}
We present the results of ongoing characterization of Cadmium Zinc Telluride (CZT) semiconductors produced by Redlen Technologies for use in X-ray astronomy. The fully fabricated hybrid detectors consist of CZT crystals with a collecting area of $2 \mathrm{~cm} \times 2 \mathrm{~cm}$ and thickness of $3 \mathrm{~mm}$ mounted on a custom ASIC originally designed for the Nuclear Spectroscopic Telescope Array (NuSTAR) mission, which launched in 2012. We present the results of electronic noise, inter-pixel conductance, and leakage current tests as well as spectral calibration using an ${ }^{241} \mathrm{Am}$ source. Despite high electronic noise due to errors in fabrication, we are able to compare characteristics of the Redlen CZT detectors to those of the CZT detectors produced by eV Products aboard NuSTAR.
\end{abstract}

Keywords: CZT, Redlen Technologies, X-ray detectors, NuSTAR, astronomy

\section{INTRODUCTION}

Cadmium Zinc Telluride (CZT) detectors are used in a variety of fields, from medical and astronomical imaging to military applications. Thanks to the high atomic numbers of its constitutive elements, CZT provides good sensitivity to hard X-rays and gamma-rays. ${ }^{1}$ In the case of X-ray astronomy, CZT detectors have been utilized successfully in several configurations. The Burst Alert Telescope (BAT) instrument on the Neil Gehrels Swift Observatory makes use of a coded-aperture mask in combination with an array of CZT detectors in order to detect and localize gamma-ray bursts (GRBs), ${ }^{2}$ while the Cadmium-Zinc-Telluride Imager (CZTI) on AstroSat ${ }^{3}$ observes GRBs and other transients using of a plane of CZT detectors with both a coded-aperture mask and a set of tantalum collimators. ${ }^{4}$

In contrast, the Nuclear Spectroscopic Telescope Array $(N u S T A R)^{5}$ observes the X-ray sky in the 3-79 keV band with two focal plane modules, each containing four CZT detectors. Each $2 \mathrm{~cm} \times 2 \mathrm{~cm} \times 2 \mathrm{~mm}$ crystal is mated to a custom pixelated ASIC via a flip-chip bonding process to produce a "hybrid." The $32 \times 32$ pixelated design of the NuSTAR hybrid allows the instrument to take advantage of the small-pixel effect, ${ }^{6}$ by which the signal induced by positively-charged holes is minimized and energy resolution is improved. In addition, this design makes the NuSTAR instrument particularly flexible in terms of application and operation, with the readout electronics providing an event list with the time, location, and energy of each photon. The pixelated architecture can however lead to charge-sharing among adjacent pixels, which adversely affects energy resolution.

Looking towards the future, we are developing the mission concept High Energy X-ray Probe $(H E X-P) .{ }^{7}$ This observatory, which will inherit much of the optics and detector design of NuSTAR, will achieve improved spatial and energy resolution in addition to an observable bandpass from $2 \mathrm{keV}$ extending as far as $200 \mathrm{keV}$. In order to realize these improvements, we have begun to develop advancements in several technologies, including focusing optics and readout electronics. In addition, we are investigating detector materials from different vendors. While NuSTAR was launched with CZT detectors from eV Products, we are currently testing Cadmium Telluride (CdTe) detectors from Acrorad Co. and CZT detectors from Redlen Technologies. The necessary qualifications for a good X-ray detector include low inter-pixel conductance in order to minimize charge sharing between adjacent pixels, low noise in the form of leakage current, and good spectral resolution. These properties should show a high degree of uniformity across a given crystal, and material from a given vendor should be relatively consistent in quality in order to reduce the time and monetary costs of testing, calibrating, and ultimately flying detectors. In this paper, we present the initial results of our ongoing characterization of CZT detectors from Redlen Technologies. Before continuing, we must include a caveat: in the process of bonding the Redlen CZT

Send correspondences to S.N.P.: spike@caltech.edu

High Energy, Optical, and Infrared Detectors for Astronomy VIII, edited by Andrew D. Holland,

James Beletic, Proc. of SPIE Vol. 10709, 107091D · C) 2018 SPIE

CCC code: $0277-786 \mathrm{X} / 18 / \$ 18 \cdot$ doi: $10.1117 / 12.2313860$

Proc. of SPIE Vol. 10709 107091D-1 
to their respective ASICs, the incorrect epoxy was used, which, as shown below, results in increased electronic noise throughout the hybrids. Nevertheless, we can still test several key performance metrics and compare the Redlen and eV material.

\section{DATA AND RESULTS}

We have performed several tests using two Redlen CZT crystals (hereafter Redlen 1 and Redlen 2) with dimensions $2 \mathrm{~cm} \times 2 \mathrm{~cm} \times 3 \mathrm{~mm}$, each attached to a $N u S T A R$ ASIC via the same flip-chip bonding procedure which was used to produce the hybrids aboard NuSTAR. We began by examining the electronic noise in the fully hybridized detectors (Sec. 2.1). This noise is not inherent to the Redlen material, but can instead be attributed to the readout electronics and hybridization process. Next, we proceeded with tests of the Redlen CZT detectors. Below, we present the methods and results of these tests, which consist of inter-pixel conductance (Sec. 2.2), leakage current (Sec. 2.3), and illumination with a radioactive source, or gamma flood (Sec. 2.4). These results are presented for two Redlen detectors and compared with results from pre-flight testing of detectors currently operating aboard NuSTAR. All measurements presented in units of energy have undergone a pixel-by-pixel gain correction. Using the gamma flood results, we determined the position in channels of the $60 \mathrm{keV}$ line emitted by a ${ }^{241} \mathrm{Am}$ source as measured by each pixel. Thus we are able to determine the $\mathrm{eV} /$ channel gain value for each pixel and convert measurements into units of energy.

\subsection{Electronic Noise}

We first determined the electronic noise present in the hybrids in order to better interpret other measurements and disentangle properties of the Redlen material from properties of the readout electronics. In order to quantify the electronic noise in a hybrid, we read out the collected charge in the capacitors of each pixel without applying a bias
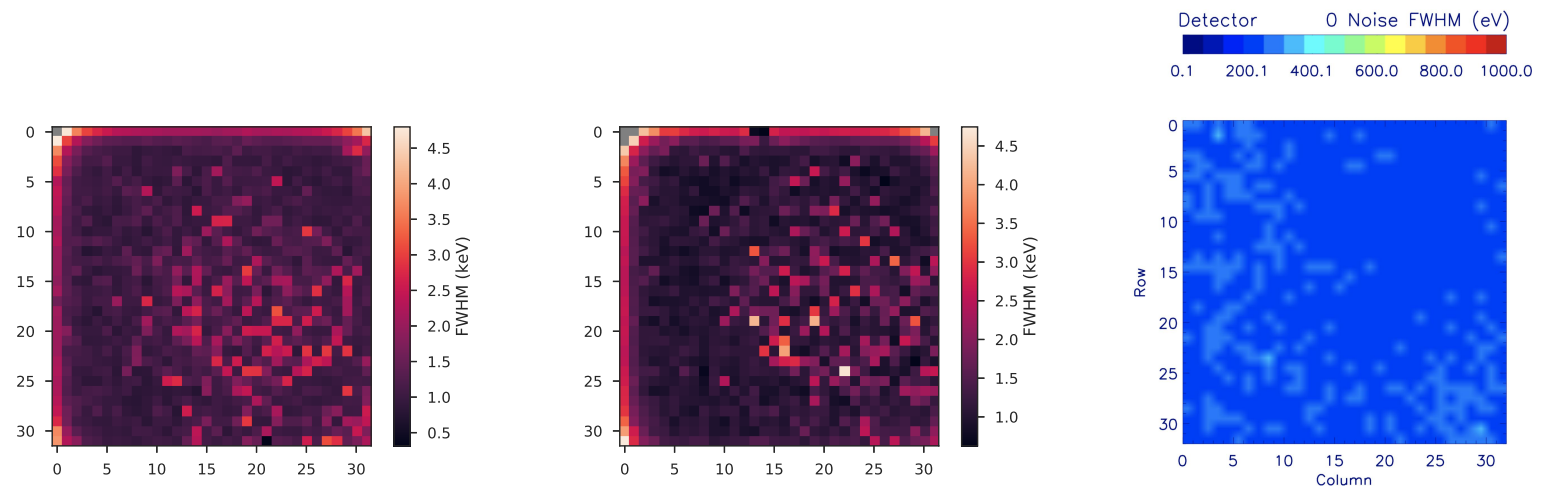

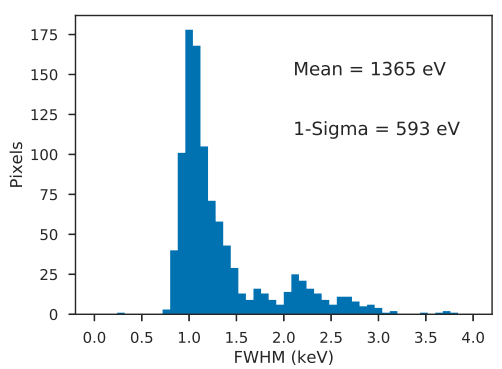

Redlen $1\left(T=-5^{\circ} \mathrm{C}\right)$

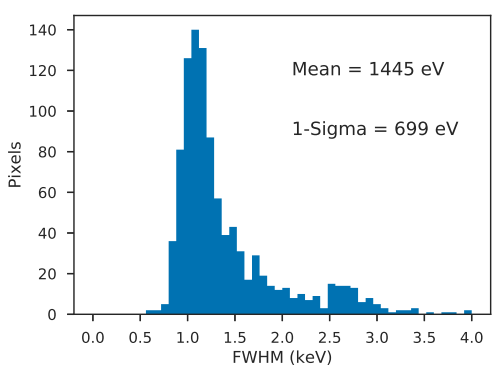

Redlen $2\left(T=-5^{\circ} \mathrm{C}\right)$
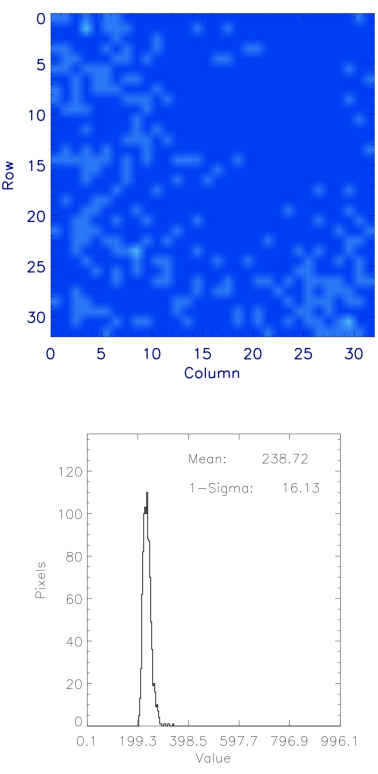

Flight $\left(T=0^{\circ} \mathrm{C}\right)$

Figure 1. Results of pixel-by-pixel electronic noise measurements for two Redlen hybrids and one flight hybrid. The top row of figures shows the FWHM of the noise distribution for each pixel. The bottom row shows the distribution of electronic noise width. We find that the Redlen hybrids have significantly higher noise than the flight hybrids. This is due to an error in the flip-chip bonding process by which the Redlen CZT detectors were attached to NuSTAR ASICs. 
voltage across the detector and without applying an artificial pulse to the pixels. The result is a distribution of charge values for each pixel which we may fit to a Gaussian, thereby determining the width of the distribution. This width is indicative of the electronic noise in the pixel. Using a Temptronic Thermostream system, we performed the electronic noise tests of the Redlen hybrids at $-5^{\circ} \mathrm{C}$. The flight hybrid which we present for comparison was tested at $0^{\circ} \mathrm{C}$. The noise measurements are presented in units of effective energy as determined by the gain correction described above.

The results of the electronic noise measurements are shown in Figure 1. We find that the Redlen hybrids exhibit very high electronic noise compared to their NuSTAR counterparts. The two Redlen hybrids we investigated each have a mean electronic noise FWHM of more than $1300 \mathrm{eV}$ - more than five times the value measured in the NuSTAR flight hybrids. In addition, the pixel-to-pixel spread of the measured electronic noise is wider in the Redlen hybrids than in the flight hybrids, with standard deviations of several hundred $\mathrm{eV}$, compared to only tens of $\mathrm{eV}$ in the flight hybrids. Relatively small populations of especially noisy pixels can be seen in both the pixel maps and the electronic noise distributions shown in Figure 1. These populations appear to lie in certain regions of the hybrids rather than being uniformly distributed.

Although the Redlen hybrids exhibit high electronic noise, we have determined that the cause of this noise is not the Redlen CZT, but the hybridization process. By examining scanning electron microscope (SEM) images of the connections between the detectors and ASICs and by measuring abnormally high resistance between the detectors and ASICs, we infer that the electronic noise arises due to the use of incorrect conducting epoxy during the flip-chip bonding process. However, we are still able to make meaningful measurements of the characteristics of Redlen Technologies' CZT material.

\subsection{Inter-pixel Conductance}

An important property of CZT detectors is the extent to which the charge is shared across adjacent pixels. Charge-sharing results in more difficult calibration as well as loss of spectral resolution. As such, inter-pixel conductance should be minimized. Due to the design of the NuSTAR hybrid, we are unable to directly probe the conductance between any given pair of adjacent pixels. Instead, we can determine the conductance across a given
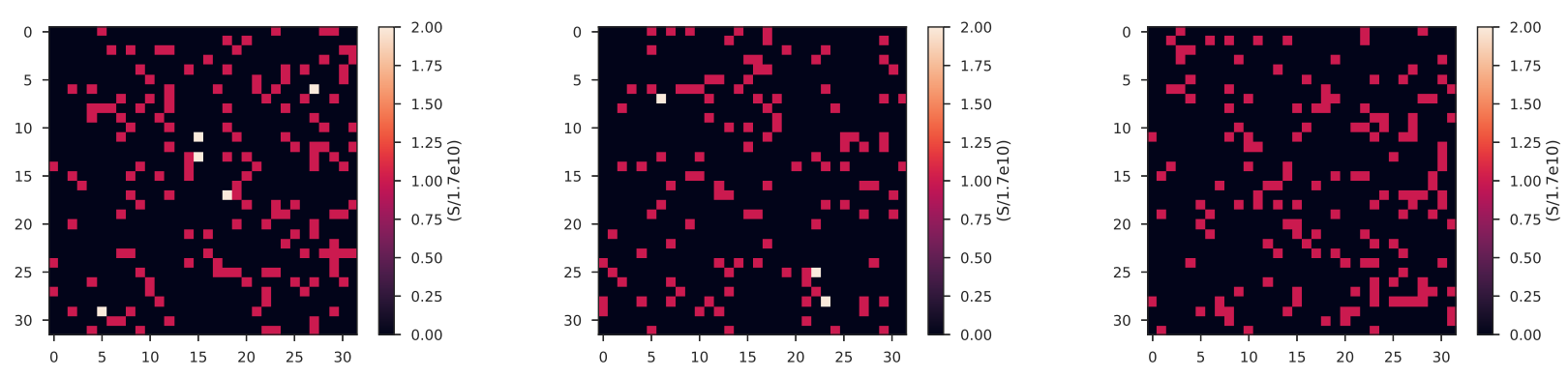

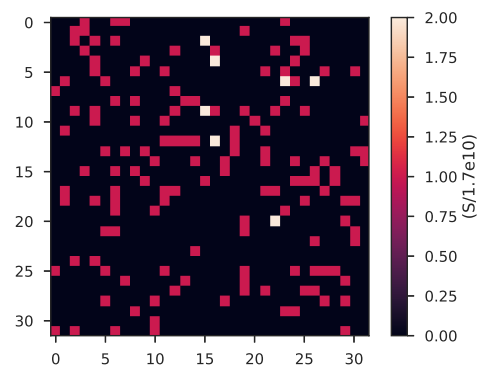

Redlen $1\left(T=-5^{\circ} \mathrm{C}\right)$

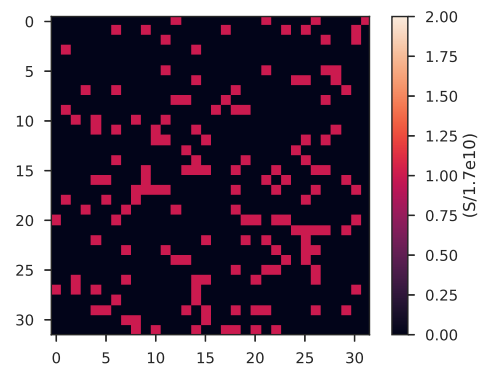

Redlen $2\left(T=-5^{\circ} \mathrm{C}\right)$

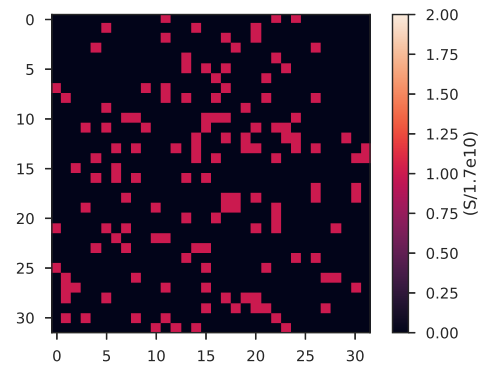

Flight $\left(T=23^{\circ} \mathrm{C}\right)$

Figure 2. Results of inter-pixel conductance measurements for two Redlen hybrids and one flight hybrid. Due to the experimental setup, each hybrid produces two conductance scans: one in the vertical direction, shown in the top row, and one in the horizontal, shown in the bottom row. 
pixel (i.e. between a pair of pixels separated by another pixel) in either the horizontal or vertical direction. Thus, we scan each row of pixels and determine the conductance across the pixels of that row. We then similarly scan each column. The result is two sets of conductance values for each pixel. We performed inter-pixel conductance scans for the two Redlen hybrids at $-5^{\circ} \mathrm{C}$, while the data presented for the flight hybrid was collected at room temperature $\left(\sim 23^{\circ} \mathrm{C}\right)$. While the flight hybrid was tested at a higher temperature than the Redlen hybrids, the conductance is not expected to change dramatically between $-5^{\circ} \mathrm{C}$ and $23^{\circ} \mathrm{C}$, and therefore we are able to compare the results.

The Redlen hybrids exhibit low conductance between pixels in both the horizontal and vertical directions, as is shown in Figure 2. The conductance of all pixels is of the order $10^{-10} \mathrm{~S}$. The inter-pixel conductance measurements of the Redlen hybrids are essentially indistinguishable from those of the flight hybrid. In particular, the conductance does not appear to vary significantly across the detectors, instead appearing uniform in both the row and column scans.

\subsection{Leakage Current}

We next investigated the leakage current of the Redlen hybrids. Leakage current is defined as the current through the detector which results from applying a bias voltage. We measured the leakage current through each pixel at several bias voltages between $100 \mathrm{~V}$ and $600 \mathrm{~V}$, and operating temperatures between $-5^{\circ} \mathrm{C}$ and $23^{\circ} \mathrm{C}$. The leakage current measured at $0 \mathrm{~V}$ is treated as a constant offset which is subtracted from the leakage current measured at higher voltages.

We find that the Redlen hybrids exhibit a lower mean leakage current than the flight hybrids at similar operating conditions. The leakage current is also uniform across the Redlen hybrids, apart from a small artifact in the lower left corner due to the experimental setup. However the variance across pixels is larger than that of
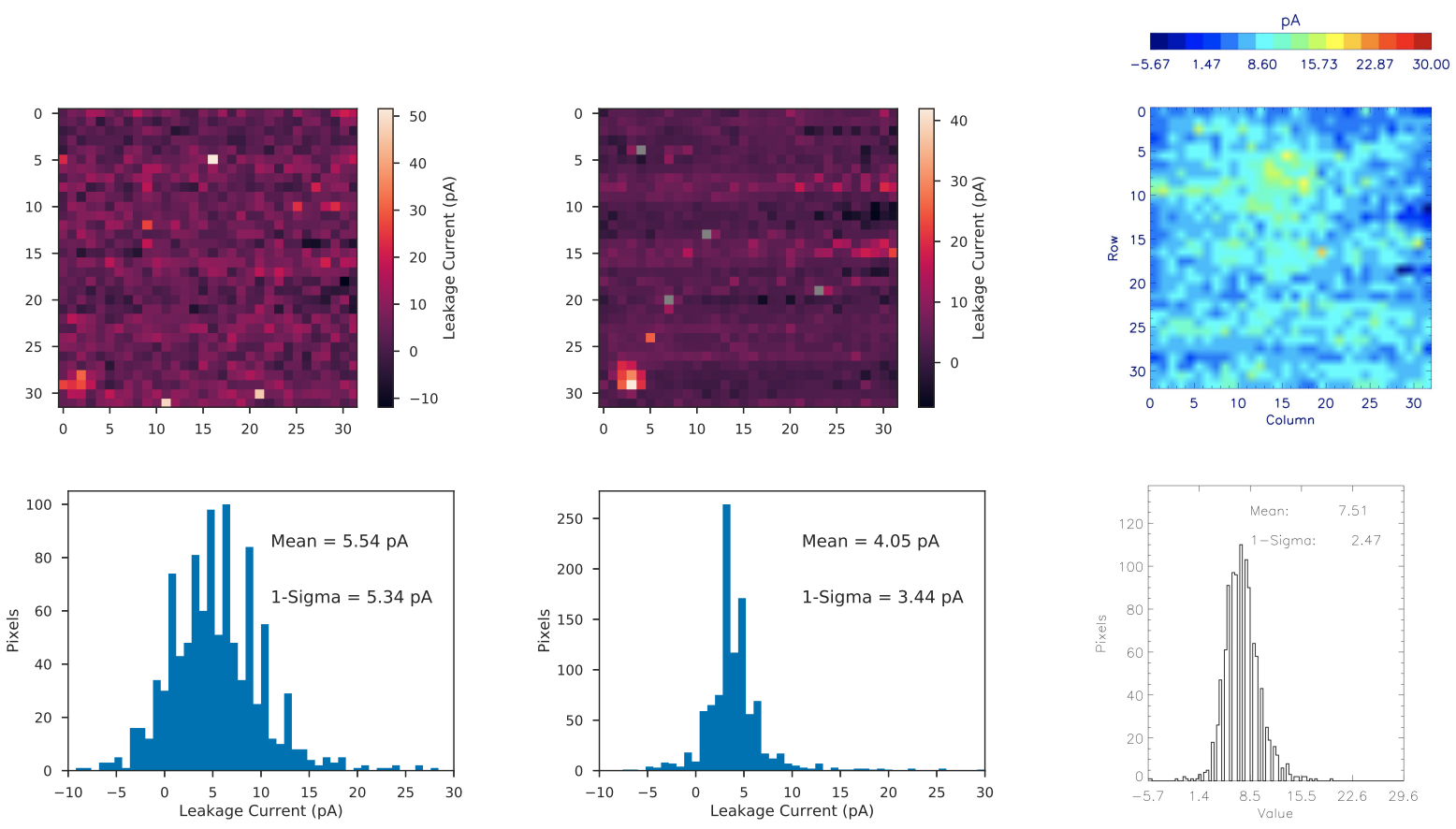

Redlen $1\left(T=5^{\circ} \mathrm{C}, V=500 \mathrm{~V}\right)$

Redlen $2\left(T=5^{\circ} \mathrm{C}, V=500 \mathrm{~V}\right)$
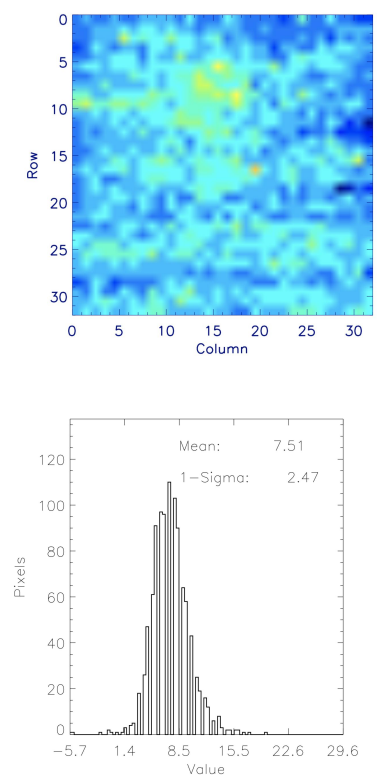

Figure 3. Results of pixel-by-pixel leakage current measurements for two Redlen hybrids and one flight hybrids. The top row of figures shows the leakage current for each pixel. Pixels shown in gray exhibit leakage current greater than 75 pA. The bottom row shows the distribution of leakage current measurements for each hybrid. We find that the Redlen hybrids have lower mean leakage current than the flight hybrids, but show more variance between pixels. 
the flight hybrids, with standard deviations comparable to the mean. In contrast, the leakage current measured in the flight hybrids exhibits less spread with a standard deviation of around one third of the mean and around half of the standard deviation measured in the Redlen hybrids. Figure 3 shows the results of the leakage current measurements of the Redlen hybrids at a bias voltage of $500 \mathrm{~V}$ and $T=5^{\circ} \mathrm{C}$. In addition, we find that at high voltages $(>400 \mathrm{~V})$, a small number of pixels begin to exhibit anomalously high leakage current $(>40 \mathrm{pA}$ at $5^{\circ} \mathrm{C}$ ). While this increases the noise at high voltage, thanks to the design of the NuSTAR ASIC we are able to turn off individual pixels so that operation at high voltage is feasible. This allows us to benefit from improved energy resolution at high voltage while minimizing adverse effects.

\subsection{Gamma Flood}

Finally, we have performed spectral tests of the Redlen hybrids. The hybrids were irradiated with an ${ }^{241} \mathrm{Am}$ source while a bias voltage of $500 \mathrm{~V}$ was applied across the detector. A temperature of $-5^{\circ} \mathrm{C}$ was maintained using during testing using the Temptronic Thermostream device. In order to determine the spectral resolution of the detectors, we determined the width of the cumulative $60 \mathrm{keV}$ line for each hybrid after applying the channelto-energy gain correction to each pixel. We have also applied identical corrections to an analogous dataset collected using a flight hybrid in order to accurately compare to the Redlen hybrids.

The results of gamma flood testing are shown in Figure 4. The Redlen hybrids produce worse spectral resolution than the flight detectors as measured by the FWHM of the cumulative ${ }^{241} \mathrm{Am} 60 \mathrm{keV}$ line. Both Redlen hybrids exhibit a line width of around $2130 \mathrm{eV}$ - more than twice the $1030 \mathrm{eV}$ FWHM measured by the flight hybrid. This poor resolution may be partly attributed to the high electronic noise in the Redlen hybrids. Like the NuSTAR flight hybrids though, the Redlen hybrids show good uniformity in terms of counts per pixel. The effects of the high electronic noise can be seen in the number of pixels which were turned off, shown in gray in Figure 4. Because the readout architecture of the NuSTAR ASIC has a limit on the number of events that
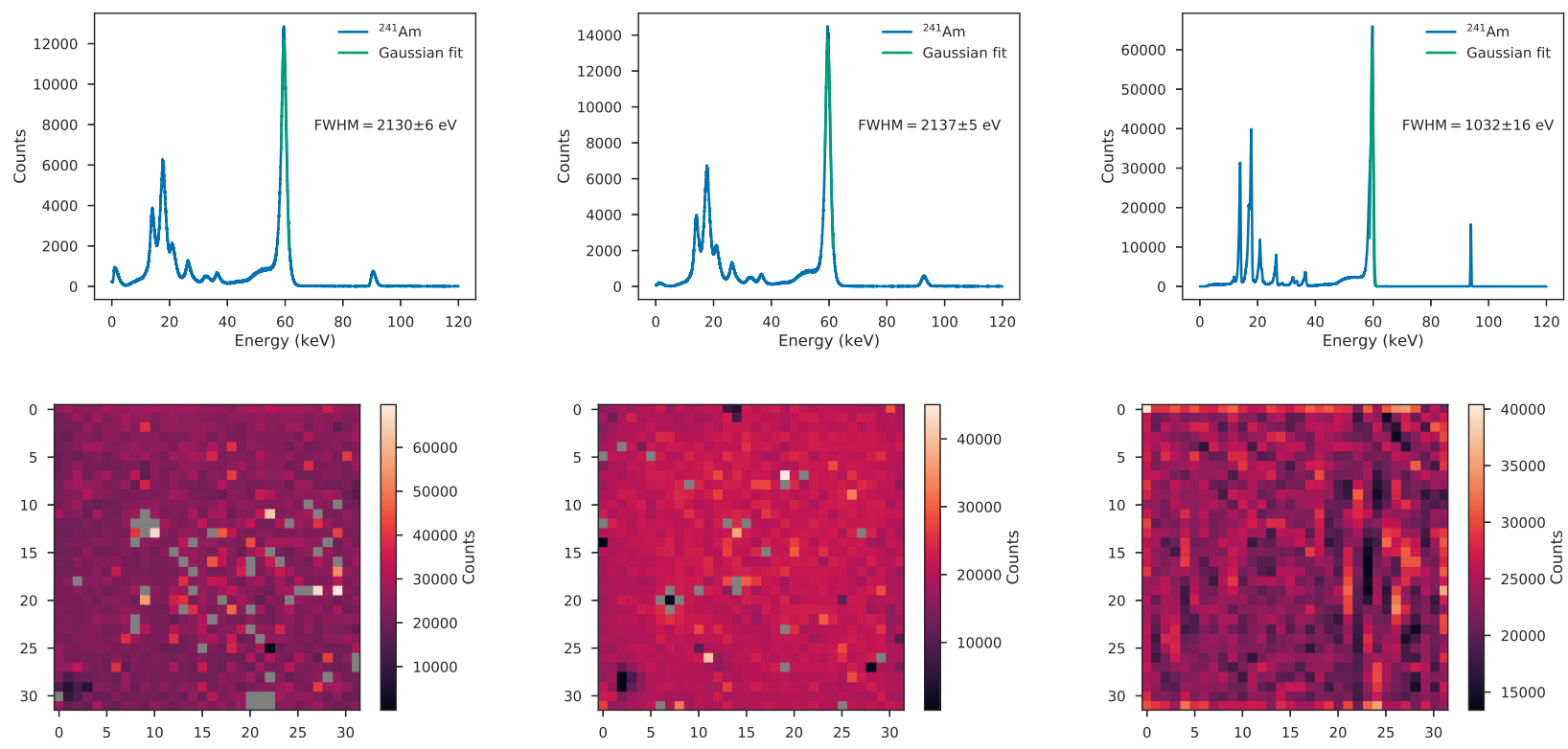

Redlen $1\left(T=-5^{\circ} \mathrm{C}, V=500 \mathrm{~V}\right)$

Redlen $2\left(T=-5^{\circ} \mathrm{C}, V=500 \mathrm{~V}\right)$

Flight $\left(T=5^{\circ} \mathrm{C}, V=400 \mathrm{~V}\right)$

Figure 4. Results of irradiating two Redlen hybrids and one flight detector with an ${ }^{241}$ Am source. The top row of figures shows the gain corrected spectra. The $60 \mathrm{keV}$ line has been fitted to a Gaussian, shown in green, to determine the width of the line as measured by each hybrid. The small line between $90 \mathrm{keV}$ and $100 \mathrm{keV}$ is due to an artificial pulse applied to the pixel at position $(10,10)$. The width of this line is an indication of the electronic noise. The bottom row of figures shows the counts per pixel for each hybrid. Pixels shown in gray were turned off during testing due to a high number of spurious events. 
can be read out in a given period of time, pixels which trigger on false events due to high noise can prevent real events from being recorded and so must be deactivated.

\section{DISCUSSION AND CONCLUSIONS}

We have performed several tests of the noise, uniformity, and resolution of two Redlen CZT detectors hybridized with NuSTAR ASICs. We found that the hybrids exhibit high electronic noise, which can be attributed to an error in the flip-chip detector-ASIC bonding process and is not due to the Redlen material. Despite this setback, we were able to collect useful information about the Redlen detectors, but due to the hybridization error our results must be considered a first look at the Redlen material and not a conclusive test of its usefulness for X-ray astronomy.

In our tests of inter-pixel conductance, leakage current, and spectral calibration, we found that the Redlen hybrids show good pixel-to-pixel uniformity, an important trait for scientific detectors. In addition, the Redlen hybrids, like those currently in use aboard $N u S T A R$, have low inter-pixel conductance, which helps to minimize charge sharing between adjacent pixels. Charge-sharing results in degraded spectral resolution, so low conductance is a good first indication of the feasibility of the Redlen CZT material. We also measure low mean leakage current compared to that present in the flight hybrids, which further drives down noise and should result in better energy resolution. However, we measure poor spectral resolution at $60 \mathrm{keV}$ which may not be completely attributable to the high electronic noise. This may be due to inhomogeneity across the detector, observed for example in the wider spread in the leakage current distributions compared to the flight detectors. Alternatively, there may be effects of the hybridization error that we have not considered which could degrade the energy resolution. We can not rule out the possibility that the Redlen material itself is the cause of the comparatively poor energy resolution, but further investigation is necessary before coming to such a conclusion. We plan to produce new hybrids using Redlen CZT in order to eliminate the high electronic noise which plagues our current results. In addition, a more thorough analysis of the characteristics of Redlen Technologies' CZT detectors is necessary. This will include an investigation of energy resolution in multiple bands, more precise gain and offset corrections, and further study of charge mobility in the Redlen material.

\section{ACKNOWLEDGMENTS}

This research was funded by NASA grant NNX13AC55G.

\section{REFERENCES}

[1] Eisen, Y. and Shor, A., "CdTe and CdZnTe materials for room-temperature X-ray and gamma ray detectors," Journal of Crystal Growth 184-185, 1302-1312 (1998).

[2] Barthelmy, S. D., "The Burst Alert Telescope (BAT) on the Swift MIDEX mission," Proceedings of SPIE The International Society for Optical Engineering 5165, 175-189 (2004).

[3] Singh, K. P., Tandon, S. N., Agrawal, P. C., Antia, H. M., Manchanda, R. K., Yadav, J. S., Seetha, S., Ramadevi, M. C., Rao, A. R., Bhattacharya, D., Paul, B., Sreekumar, P., Bhattacharyya, S., Stewart, G. C., Hutchings, J., Annapurni, S. A., Ghosh, S. K., Murthy, J., Pati, A., Rao, N. K., Stalin, C. S., Girish, V., Sankarasubramanian, K., Vadawale, S., Bhalerao, V. B., Dewangan, G. C., Dedhia, D. K., Hingar, M. K., Katoch, T. B., Kothare, A. T., Mirza, I., Mukerjee, K., Shah, H., Shah, P., Mohan, R., Sangal, A. K., Nagabhusana, S., Sriram, S., Malkar, J. P., Sreekumar, S., Abbey, A. F., Hansford, G. M., Beardmore, A. P., Sharma, M. R., Murthy, S., Kulkarni, R., Meena, G., Babu, V. C., and Postma, J., "ASTROSAT mission," in [Proceedings of SPIE], 9144 (2014).

[4] Bhalerao, V., Bhattacharya, D., Vibhute, A., Pawar, P., Rao, A. R., Hingar, M. K., Khanna, R., Kutty, A. P., Malkar, J. P., Patil, M. H., Arora, Y. K., Sinha, S., Priya, P., Samuel, E., Sreekumar, S., Vinod, P., Mithun, N. P., Vadawale, S. V., Vagshette, N., Navalgund, K. H., Sarma, K. S., Pandiyan, R., Seetha, S., and Subbarao, K., "The Cadmium Zinc Telluride Imager on AstroSat," Journal of Astrophysics and Astronomy 38(2) (2017). 
[5] Harrison, F. A., Craig, W. W., Christensen, F. E., Hailey, C. J., Zhang, W. W., Boggs, S. E., Stern, D., Cook, W. R., Forster, K., Giommi, P., Grefenstette, B. W., Kim, Y., Kitaguchi, T., Koglin, J. E., Madsen, K. K., Mao, P. H., Miyasaka, H., Mori, K., Perri, M., Pivovaroff, M. J., Puccetti, S., Rana, V. R., Westergaard, N. J., Willis, J., Zoglauer, A., An, H., Bachetti, M., Barriere, N. M., Bellm, E. C., Bhalerao, V., Brejnholt, N. F., Fuerst, F., Liebe, C. C., Markwardt, C. B., Nynka, M., Vogel, J. K., Walton, D. J., Wik, D. R., Alexander, D. M., Cominsky, L. R., Hornschemeier, A. E., Hornstrup, A., Kaspi, V. M., Madejski, G. M., Matt, G., Molendi, S., Smith, D. M., Tomsick, J. a., Ajello, M., Ballantyne, D. R., Balokovic, M., Barret, D., Bauer, F. E., Blandford, R. D., Brandt, W. N., Brenneman, L. W., Chiang, J., Chakrabarty, D., Chenevez, J., Comastri, A., Elvis, M., Fabian, A. C., Farrah, D., Fryer, C. L., Gotthelf, E. V., Grindlay, J. E., Helfand, D. J., Krivonos, R., Meier, D. L., Miller, J. M., Natalucci, L., Ogle, P., Ofek, E. O., Ptak, A., Reynolds, S. P., Rigby, J. R., Tagliaferri, G., Thorsett, S. E., Treister, E., and Urry, C. M., "The Nuclear Spectroscopic Telescope Array (NuSTAR) Mission," The Astrophysical Journal 770(1), 20 (2013).

[6] Zumbiehl, A., Hage-Ali, M., Fougeres, P., Koebel, J. M., Regal, R., Rit, C., Ayoub, M., and Siffert, P., "Modelling and 3D optimisation of CdTe pixels detector array geometry - Extension to small pixels," Nuclear Instruments and Methods in Physics Research, Section A: Accelerators, Spectrometers, Detectors and Associated Equipment 469(2), 227-239 (2001).

[7] Harrison, F. A., Madsen, K. K., Grefenstette, B. W., Stern, D. K., Hornschmeier, A. E., Boggs, S. E., Brandt, N., Brenneman, L., Chakrabarty, D., Christensen, F. E., Craig, W., Fabian, A. C., Elvis, M., Grindlay, J., Hailey, C., Hornstrup, A., Kaspi, V., Madjeski, G., Matt, G., Miller, J. M., Miyasaka, H., Molendi, S., Zhang, W. W., Tomsick, J., and Yukita, M., "The high-energy x-ray probe (HEX-P)," in [SPIE Astronomical Telescopes and Instrumentation], (2018). 